\title{
ON LAGRANGE INTERPOLATION AT DISTURBED ROOTS OF UNITY
}

\author{
CHARLES K. CHUI, XIE-CHANG SHEN, AND LEFAN ZHONG
}

\begin{abstract}
Let $z_{n k}=e^{i t_{n k}}, 0 \leq t_{n 0}<\cdots<t_{n n}<2 \pi, f$ a function in the disc algebra $A$, and $\mu_{n}=\max \left\{\left|t_{n k}-2 k \pi /(n+1)\right|: 0 \leq k \leq n\right\}$. Denote by $L_{n}(f ; \cdot)$ the polynomial of degree $n$ that agrees with $f$ at $\left\{z_{n k}\right.$ : $k=0, \ldots, n\}$. In this paper, we prove that for every $p, 0<p<\infty$, there exists a $\delta_{p}>0$, such that $\left\|L_{n}(f ; \cdot)-f\right\|_{p}=O\left(\omega\left(f ; \frac{1}{n}\right)\right)$ whenever $\mu_{n} \leq \delta_{p} /(n+1)$. It must be emphasized that $\delta_{p}$ necessarily depends on $p$, in the sense that there exists a family $\left\{z_{n k}: k=0, \ldots, n\right\}$ with $\mu_{n}=\delta_{2} /(n+1)$ and such that $\left\|L_{n}(f ; \cdot)-f\right\|_{2}=O\left(\omega\left(f ; \frac{1}{n}\right)\right)$ for all $f \in A$, but $\sup \left\{\left\|L_{n}(f ; \cdot)\right\|_{p}: f \in A,\|f\|_{\infty}=1\right\}$ diverges for sufficiently large values of $p$. In establishing our estimates, we also derive a Marcinkiewicz-Zygmund type inequality for $\left\{z_{n k}\right\}$.
\end{abstract}

\section{INTRODUCTION}

Let $D$ be the open unit disc in the complex plane with closure $\bar{D}$ and boundary $T$. Also, let $z_{n k}=e^{i t_{n k}}, 0 \leq t_{n 0}<\cdots<t_{n n}<2 \pi$, and for a function $f$ defined on $T$, let $L_{n}(f ; \cdot)$ be the Lagrange polynomial of degree $n$ that interpolates $f$ at $\left\{z_{n k}: k=0, \ldots, n\right\}$. If $f$ is analytic on $\bar{D}$, then the following result is well known (cf. [17, Chapter 7]).

Theorem A. For any $f$ analytic on $\bar{D}$, a necessary and sufficient condition for

$$
\left\|L_{n}(f ; \cdot)-f\right\|_{\infty} \rightarrow 0
$$

is that the family $\left\{z_{n k}: k=0, \ldots, n\right\}$ is uniformly distributed on $T$.

Here and throughout, we use the usual notation:

$$
\|f\|_{p}= \begin{cases}\left\{\frac{1}{2 \pi} \int_{0}^{2 \pi}\left|f\left(e^{i \theta}\right)\right|^{p} d \theta\right\}^{\frac{1}{p}} & \text { for } 0<p<\infty, \\ \sup _{|z|=1}|f(z)| & \text { for } p=\infty .\end{cases}
$$

Received by the editors April 26, 1990 and, in revised form, January 10, 1991.

1991 Mathematics Subject Classification. Primary 41A05; Secondary 30A10, 41A10.

Key words and phrases. Disturbed roots of unity, Marcinkiewicz-Zygmund type inequality, Lagrange interpolation, order of approximation, $A_{p}$-weights, $H^{p}$-interpolation.

The first author was supported by NSF Grants DMS-89-01345 and INT-87-12424, and SDIO/IST managed by ARO under contract DAAL 03-90-G-0091.

The second author was supported by NSFC and the Chinese National Commission of Education. 
In this paper, we consider Lagrange interpolation of functions in the disc algebra $A$; that is, functions which are analytic in $D$ and continuous on $\bar{D}$. It is well known, however, that the above result does not hold for $f \in A$ in general (cf. [6, 16]). In fact, Vértesi [16] proved in 1982 that for any family $\left\{z_{n k}: k=0, \ldots, n\right\}$, there exists an $f_{0} \in A$ such that

$$
\limsup _{n \rightarrow \infty}\left|L_{n}\left(f_{0} ; \cdot\right)\right|=\infty
$$

almost everywhere on $T$. For this reason, we must consider convergence in $L_{p}=L_{p}(T), 0<p<\infty$. In this direction, probably the earliest result is due to Lozinski [10] in 1941, as follows.

Theorem B. For $0<p<\infty$ and $z_{n k}=\exp (i 2 k \pi /(n+1))$,

$$
\left\|L_{n}(f ; \cdot)-f\right\|_{p} \rightarrow 0
$$

for all $f \in A$.

Related results on interpolation at the roots of unity have also been obtained by Walsh and Sharma [18], Sharma and Vértesi [13], Saff and Walsh [12], and Shen [14], and at Fejér points on a Jordan curve by Curtiss [4], $\mathrm{Al}^{\prime}$ per and Kalinogorskaja [1], Shen and Zhong [15], and Chui and Shen [2]. In this paper, we consider sample points $z_{n k}$ which are not necessarily the $(n+1)$ th roots of unity.

In order to give a sharp estimate of the order of convergence, we first establish the following Marcinkiewicz-Zygmund type inequality which is of independent interest. To facilitate our presentation, we need the following notation:

$$
\pi_{n}=\text { the class of polynomials of degree at most } n
$$

and

$$
\mu_{n}=\max _{0 \leq k \leq n}\left|t_{n k}-\frac{2 k \pi}{n+1}\right| .
$$

Theorem 1. For any $p, 1<p<\infty$, there exist positive constants $\delta_{p}$ and $C_{p}$, such that whenever

$$
\mu_{n} \leq \frac{\delta_{p}}{n+1}
$$

then

$$
\frac{1}{2 \pi} \int_{0}^{2 \pi}\left|P_{n}\left(e^{i \theta}\right)\right|^{p} d \theta \leq \frac{C_{p}}{n+1} \sum_{k=0}^{n}\left|P_{n}\left(z_{n k}\right)\right|^{p}
$$

for all $P_{n} \in \pi_{n}$, where $z_{n k}=e^{i t_{n k}}$.

As usual, $E_{n}(f)$ will denote the error of uniform approximation of $f$ on $T$ from $\pi_{n}$, namely:

$$
E_{n}(f)=\inf _{q \in \pi_{n}}\|f-q\|_{\infty} .
$$

The main theorem in this paper is the following. 
Theorem 2. For any $p, 0<p<\infty$, there exist positive constants $\delta_{p}$ and $C_{p}^{\prime}$, such that whenever (1.2) is satisfied, then

$$
\left\|L_{n}(f ; \cdot)-f\right\|_{p} \leq C_{p}^{\prime} E_{n}(f)
$$

for any $f \in A$.

We remark that for $1<p<\infty$, the $\delta_{p}$ in the above two theorems are the same, and for $0<p \leq 1$, we may choose $\delta_{p}=\delta_{p^{\prime}}$ for any $p^{\prime}>1$. As a simple consequence of the above theorem, we have the following result.

Corollary 1. Under the hypotheses stated in Theorem 2,

$$
\left\|L_{n}(f ; \cdot)-f\right\|_{p} \leq C_{p, r} n^{-r} \omega\left(f^{(r)} ; \frac{1}{n}\right)
$$

for any $f \in A$ with $f\left(e^{i t}\right) \in C^{r}[0,2 \pi]$, where $\omega(g ; t)$ is the uniform modulus of continuity of $g$ and $C_{p, r}$ is an absolute constant, depending only on $p$ and $r$.

We remark that in condition (1.2) the constant $\delta_{p}$ must necessarily depend on $p$. In $\S 4$, we will construct a family $\left\{z_{n k}: k=0, \ldots, n\right\}$ with $\mu_{n} \leq$ $\delta /(n+1)$, where $\delta>0$, for which

$$
\left\|L_{n}(f ; \cdot)-f\right\|_{2} \leq C_{2} E_{n}(f)
$$

for all $f \in A$, but

$$
\sup \left\{\left\|L_{n}(f ; \cdot)\right\|_{p}: f \in A,\|f\|_{\infty}=1\right\} \rightarrow \infty .
$$

We also remark that a necessary condition for $\left\|L_{n}(f ; \cdot)-f\right\|_{p} \rightarrow 0$, where $f \in A$ and $0<p<\infty$, is that the family $\left\{z_{n k}: k=0, \ldots, n\right\}$ is uniformly distributed on $T$. This will be proved in $\S 5$. (Recall from Theorem A that this is also a necessary condition for $\left\|L_{n}(f ; \cdot)-f\right\|_{\infty} \rightarrow 0$.)

\section{Preliminary Results}

We first derive a result in harmonic analysis which is perhaps of independent interest. Let $\varphi \in L^{1}(T)$. Recall that $\varphi \in$ BMO with norm $\|\varphi\|_{*}$ if

$$
\|\varphi\|_{*}:=\sup _{I} \frac{1}{|I|} \int_{I}\left|\varphi\left(e^{i \theta}\right)-\varphi_{I}\right| d \theta<\infty,
$$

where the supremum is taken over all arcs $I$ on $T$ with length $|I|$ and

$$
\varphi_{I}=\frac{1}{|I|} \int_{I} \varphi\left(e^{i \theta}\right) d \theta .
$$

The following is a well-known result due to John-Nirenberg (cf. [8, Chapter 6]).

Theorem C. There exist positive constants $m$ and $M$ such that for any $\varphi \in$ $B M O$, any arc $I \subset T$, and any $\lambda>0$,

$$
\frac{\left|\left\{e_{i \theta} \in I:\left|\varphi\left(e^{i \theta}\right)-\varphi_{I}\right|>\lambda\right\}\right|}{|I|} \leq M \exp \left\{\frac{-m \lambda}{\|\varphi\|_{*}}\right\} .
$$

We remark that the constants $m$ and $M$ can be chosen to be $m=\frac{1}{4 e}$ and $M$ $=\sqrt{e}$.

The following result on the BMO norm can be found in [9]. 
Theorem D. There exists an absolute constant $C_{*}$ such that for any $g\left(e^{i \theta}\right) \in$ $B M O$ with $g(z)$ analytic in $|z|>1$ and bounded at $\infty$,

$$
\left\|g\left(e^{i \theta}\right)\right\|_{*} \leq C_{*} \inf \left\{\|g-h\|_{\infty}: h \in H^{\infty}\right\} .
$$

For any $\delta>0$ and $1<p<\infty$, a nonnegative function $w$ defined on $T$ is called an $A_{p}$-weight relative to $\delta$ if

$$
\sup _{I}\left(\frac{1}{|I|} \int_{I} w\left(e^{i \theta}\right) d \theta\right)\left(\frac{1}{|I|} \int_{I}\left(w\left(e^{i \theta}\right)\right)^{-\frac{1}{p-1}} d \theta\right)^{p-1}<\delta .
$$

For $A_{p}$-weights, the following result is due to Muckenhoupt [11].

Theorem E. Let $1<p<\infty$ and $w\left(e^{i \theta}\right)$ be an $A_{p}$-weight relative to some $\delta>0$. Then for any $g \in L^{p}(T)$, its Cauchy transform

$$
(H g)(z):=\frac{1}{2 \pi i} \int_{T} \frac{g(\zeta)}{\zeta-z} d \zeta, \quad|z|<1
$$

satisfies

$$
\int_{0}^{2 \pi}\left|(H g)\left(e^{i \theta}\right)\right|^{p} w\left(e^{i \theta}\right) d \theta \leq C_{H} \int_{0}^{2 \pi}\left|g\left(e^{i \theta}\right)\right|^{p} w\left(e^{i \theta}\right) d \theta,
$$

where $C_{H}$ is an absolute constant depending only on $\delta$ and $p$.

Of course, $(H g)\left(e^{i \theta}\right)$ in inequality (2.3) is the almost everywhere radial limit of $(\mathrm{Hg})\left(r e^{i \theta}\right)$.

We have the following result.

Lemma 1. For any $p, 1<p<\infty$, there exists an $\varepsilon_{p}>0$, such that for all $\varphi \in B M O$ with $\|\varphi\|_{*}<\varepsilon_{p},\left|e^{\varphi}\right|$ is an $A_{p}$-weight relative to $\delta=2$.

Proof. Since $\|\operatorname{Re} \varphi\|_{*} \leq\|\varphi\|_{*}$, we may assume, without loss of generality, that $\varphi$ is a real-valued function. Set $\psi=-\varphi /(p-1)$ and $w=e^{\varphi}$. Then we have

$$
\begin{aligned}
& \left(\frac{1}{|I|} \int_{I} w d \theta\right)\left(\frac{1}{|I|} \int_{I} w^{-\frac{1}{p-1}} d \theta\right)^{p-1} \\
& \quad=\left(\frac{1}{|I|} \int_{I} e^{\varphi-\varphi_{I}} d \theta\right)\left(\frac{1}{|I|} \int_{I} e^{\psi-\psi} d \theta\right)^{p-1},
\end{aligned}
$$

where it follows from Theorem $\mathrm{C}$ that

$$
\begin{aligned}
\frac{1}{|I|} \int_{I} e^{\varphi-\varphi_{I}} d \theta & =\frac{1}{|I|} \int_{0}^{\infty}\left|\left\{z \in I: e^{\varphi(z)-\varphi_{I}}>\lambda\right\}\right| d \lambda \\
& \leq \frac{1}{|I|} \int_{0}^{\infty}\left|\left\{z \in I:\left|\varphi(z)-\varphi_{I}\right|>\ln \lambda\right\}\right| d \lambda \\
& \leq 1+\frac{1}{|I|} \int_{1}^{\infty}\left|\left\{z \in I:\left|\varphi(z)-\varphi_{I}\right|>\ln \lambda\right\}\right| d \lambda \\
& \leq 1+\int_{1}^{\infty} M \exp \left\{-\frac{m \ln \lambda}{\|\varphi\|_{*}}\right\} d \lambda=1+\frac{M\|\varphi\|_{*}}{m-\|\varphi\|_{*}}
\end{aligned}
$$

Similarly, we also have

$$
\frac{1}{|I|} \int_{I} e^{\psi-\psi_{I}} d \theta \leq 1+\frac{M\|\psi\|_{*}}{m-\|\psi\|_{*}}=1+\frac{M\|\varphi\|_{*}}{(p-1) m-\|\varphi\|_{*}} .
$$


Combining these two estimates in (2.4), we have

$$
\begin{aligned}
& \left(\frac{1}{|I|} \int_{I} w d \theta\right)\left(\frac{1}{|I|} \int_{I} w^{-\frac{1}{p-1}} d \theta\right)^{p-1} \\
& \quad \leq\left(1+\frac{M\|\varphi\|_{*}}{m-\|\varphi\|_{*}}\right)\left(1+\frac{M\|\varphi\|_{*}}{(p-1) m-\|\varphi\|_{*}}\right)^{p-1}<2
\end{aligned}
$$

for all $\varphi \in \mathrm{BMO}$ with sufficiently small $\|\varphi\|_{*}$.

Next, to go from $T$ to $D$, we introduce the function

$$
\omega_{n}(z)=\prod_{k=0}^{n}\left(z-\left(1-\frac{1}{n+1}\right) z_{n k}\right),
$$

where $z_{n k}=e^{i t_{n k}}$. Our key lemma for establishing Theorem 1 is the following.

Lemma 2. Let $1<p<\infty$ and $\mu_{n}$ be defined as in (1.1). Then there exists a positive constant $\delta_{p}$ such that whenever $\mu_{n} \leq \delta_{p} /(n+1),\left|\omega_{n}\left(e^{i \theta}\right)\right|^{p}$ is an $A_{p}$-weight relative to $2^{p+1}$.

Proof. We introduce three more polynomials:

$$
\left\{\begin{array}{l}
\omega_{n}^{*}(z)=\prod_{k=0}^{n}\left(z-\left(1+\frac{1}{n+1}\right) z_{n k}\right) \\
\tilde{\omega}_{n}(z)=z^{n+1}-\left(1-\frac{1}{n+1}\right)^{n+1} \\
\tilde{\omega}_{n}^{*}(z)=z^{n+1}-\left(1+\frac{1}{n+1}\right)^{n+1}
\end{array}\right.
$$

Clearly, $\ln \left(\frac{\omega_{n}(z)}{\tilde{\omega}_{n}(z)}\right)$ is in BMO on $T$, analytic in $|z|>1$, and bounded at $\infty$, so that it follows from Theorem $D$ that

$$
\begin{aligned}
& \left\|\ln \left(\frac{\omega_{n}\left(e^{i \theta}\right)}{\tilde{\omega}_{n}\left(e^{i \theta}\right)}\right)\right\|_{*} \leq C_{*}\left\|\ln \left(\frac{\omega_{n}(z)}{\tilde{\omega}_{n}(z)}\right)-\ln \left(\frac{\omega_{n}^{*}(z)}{\tilde{\omega}_{n}^{*}(z)}\right)\right\|_{\infty} \\
& =C_{*} \max _{\theta}\left|\sum_{k=0}^{n} \ln \frac{\left(e^{i \theta}-\left(1-\frac{1}{n+1}\right) e^{i t_{n k}}\right)\left(e^{i \theta}-\left(1+\frac{1}{n+1}\right) e^{i \frac{2 k \pi}{n+1}}\right)}{\left(e^{i \theta}-\left(1+\frac{1}{n+1}\right) e^{i t_{n k}}\right)\left(e^{i \theta}-\left(1-\frac{1}{n+1}\right) e^{i \frac{2 k \pi}{n+1}}\right)}\right| \\
& =C_{*} \max _{\theta}\left|\sum_{k=0}^{n} \ln \left(1+\frac{2 e^{i \theta}\left(e^{i t_{n k}}-e^{i \frac{2 k \pi}{n+1}}\right)}{(n+1)\left(e^{i \theta}-\left(1+\frac{1}{n+1}\right) e^{i t_{n k}}\right)\left(e^{i \theta}-\left(1-\frac{1}{n+1}\right) e^{i \frac{2 k \pi}{n+1}}\right)}\right)\right| .
\end{aligned}
$$

To estimate the quantity

$$
I_{n}(\theta):=\frac{2 e^{i \theta}\left(e^{i t_{n k}}-e^{i \frac{2 k \pi}{n+1}}\right)}{(n+1)\left(e^{i \theta}-\left(1+\frac{1}{n+1}\right) e^{i t_{n k}}\right)\left(e^{i \theta}-\left(1-\frac{1}{n+1}\right) e^{i \frac{2 k \pi}{n+1}}\right)}
$$

on $[0,2 \pi]$, it is sufficient to consider $0 \leq \theta \leq \frac{\pi}{n+1}$. We separate the estimation of the denominator of $I_{n}(\theta)$ in (2.9) into two cases: 
(i) For $k=0$ and $0 \leq \theta \leq \frac{\pi}{n+1}$, we have

$$
\left|e^{i \theta}-\left(1+\frac{1}{n+1}\right) e^{i t_{n k}}\right| \geq\left(1+\frac{1}{n+1}\right)-1=\frac{1}{n+1} .
$$

(ii) For $k \geq 1$ and $0 \leq \theta \leq \frac{\pi}{n+1}$, we assume, without loss of generality, that $\delta_{p} \leq \frac{1}{30}$, so that

$$
\left|t_{n k}-\frac{2 k \pi}{n+1}\right| \leq \frac{1}{30(n+1)}, \quad k=1, \ldots, n .
$$

Hence, it follows that, for $k \geq 1$,

$$
\begin{aligned}
\mid e^{i \theta}-\left(1+\frac{1}{n+1}\right) e^{i t_{n k} \mid} & \geq\left|e^{i \theta}-e^{i t_{n k}}\right|-\frac{1}{n+1} \geq \frac{2}{\pi}\left|\theta-t_{n k}\right|-\frac{1}{n+1} \\
& \geq \frac{2}{\pi}\left(\left|\theta-\frac{2 k \pi}{n+1}\right|-\left|\frac{2 k \pi}{n+1}-t_{n k}\right|\right)-\frac{1}{n+1} \\
& \geq \frac{2}{\pi}\left(\frac{(2 k-1) \pi}{n+1}-\frac{1}{30(n+1)}\right)-\frac{1}{n+1} \\
& \geq \frac{4 k}{3(n+1)} .
\end{aligned}
$$

Combining the estimates in (i) and (ii), we have

$$
\left|e^{i \theta}-\left(1+\frac{1}{n+1}\right) e^{i t_{n k}}\right| \geq \frac{2(k+1)}{3(n+1)}, \quad k=0, \ldots, n .
$$

The same lower bound also applies to the quantity

$$
\left|e^{i \theta}-\left(1-\frac{1}{n+1}\right) e^{i \frac{2 k \pi}{n+1}}\right| .
$$

Consequently, we have the following estimate of $I_{n}(\theta)$ defined in (2.9):

$$
\begin{aligned}
\left|I_{n}(\theta)\right| & \leq \frac{9}{2} \cdot \frac{n+1}{(k+1)^{2}}\left|e^{i t_{n k}}-e^{i \frac{2 k \pi}{n+1}}\right| \\
& \leq \frac{9}{2} \cdot \frac{n+1}{(k+1)^{2}}\left|t_{n k}-\frac{2 k \pi}{n+1}\right| \leq \frac{9}{2} \cdot \frac{n+1}{(k+1)^{2}} \mu_{n} .
\end{aligned}
$$

Recalling that $\delta_{p} \leq \frac{1}{30}$, we have, for $\mu_{n} \leq \frac{\delta_{p}}{n+1}$,

$$
\left|I_{n}(\theta)\right| \leq \frac{9}{2} \frac{n+1}{(k+1)^{2}} \mu_{n} \leq \frac{3}{20}<\frac{2}{3}, \quad k=0, \ldots, n .
$$

Note that for $|\zeta| \leq \frac{2}{3}$, we have $|\ln (1+\zeta)| \leq 2|\zeta|$, so that by using $\zeta=I_{n}(\theta)$ and estimate (2.10), the result in (2.8) yields

$$
\left\|\ln \left(\frac{\omega_{n}\left(e^{i \theta}\right)}{\tilde{\omega}_{n}\left(e^{i \theta}\right)}\right)\right\|_{*} \leq 9 C_{*}(n+1) \mu_{n} \sum_{K=0}^{n} \frac{1}{(k+1)^{2}}<18 C_{*}(n+1) \mu_{n} .
$$

Hence, it follows from Lemma 1 that if 


$$
\mu_{n} \leq \frac{\varepsilon_{p}}{18 C_{*} p(n+1)},
$$

then $\left|\frac{\omega_{n}\left(e^{i \theta}\right)}{\tilde{\omega}_{n}\left(e^{i \theta}\right)}\right|^{p}$ is an $A_{p}$-weight relative to $\delta=2$. However, it is clear that since

$$
2^{-p} \leq\left|\tilde{\omega}_{n}\left(e^{i \theta}\right)\right|^{p} \leq 2^{p},
$$

$\left|\omega_{n}\left(e^{i \theta}\right)\right|^{p}$ is also an $A_{p}$-weight relative to $\delta=2^{p+1}$. In view of (2.11), this completes the proof of Lemma 2 by choosing

$$
\delta_{p}=\min \left(\frac{\varepsilon_{p}}{18 C_{*} p}, \frac{1}{30}\right) .
$$

In what follows, we need a result on $H^{p}$-interpolation. As usual, a sequence $\left\{\zeta_{j}\right\}, j=1,2, \ldots$, in $D$ is said to be $\delta$-uniformly separated, where $\delta>0$, if

$$
\prod_{\substack{j=1 \\ j \neq k}}^{\infty}\left|\frac{\zeta_{j}-\zeta_{k}}{1-\bar{\zeta}_{k} \zeta_{j}}\right| \geq \delta>0 \quad \text { all } k .
$$

The following result can be found in [5, Chapter 9, p. 149].

Theorem F. Let $0<p<\infty$ and $\left\{\zeta_{j}\right\}$ be a $\delta$-uniformly separated sequence in $D$. Then for any sequence of complex numbers $\left\{a_{j}\right\}$ satisfying

$$
\sum_{j=1}^{\infty}\left|a_{j}\right|^{p}\left(1-\left|\zeta_{j}\right|^{2}\right)<\infty
$$

there exists a function $g \in H^{p}$, such that

(i) $g\left(\zeta_{j}\right)=a_{j}, j=1,2, \ldots$, and

(ii)

$$
\frac{1}{2 \pi} \int_{0}^{2 \pi}\left|g\left(e^{i \theta}\right)\right|^{p} d \theta \leq C_{p, \delta} \sum_{j=1}^{\infty}\left|a_{j}\right|^{p}\left(1-\left|\zeta_{j}\right|^{2}\right)
$$

where $C_{p, \delta}$ is an absolute constant depending only on $p$ and $\delta$.

We remark that the above theorem holds for any finite sequence, provided that $\delta$ is independent of the length of this sequence. In order to apply the above theorem, we need the following.

Lemma 3. Let $\mu_{n}$ satisfy (1.2). Then the sequence $\left\{\left(1-\frac{1}{n+1}\right) e^{i t_{n k}}\right\}, k=$ $0, \ldots, n$, is $\delta$-uniformly separated for some $\delta>0$ independent of $n$.

Proof. To simplify the notation, set

$$
\rho=\left(1-\frac{1}{n+1}\right) \text { and } \zeta_{k}=\rho z_{n k}=\left(1-\frac{1}{n+1}\right) e^{i t_{n k}},
$$

where $z_{n k}=e^{i t_{n k}}$. Then

$$
\begin{aligned}
\left|\frac{\zeta_{j}-\zeta_{k}}{1-\bar{\zeta}_{k} \zeta_{j}}\right|^{2} & =\frac{2 \rho^{2}\left(1-\cos \left(t_{n j}-t_{n k}\right)\right)}{1-2 \rho^{2} \cos \left(t_{n j}-t_{n k}\right)+\rho^{4}} \\
& =\frac{4 \rho^{2} \sin ^{2}\left(\frac{t_{n j}-t_{n k}}{2}\right)}{\left(1-\rho^{2}\right)^{2}+4 \rho^{2} \sin ^{2}\left(\frac{t_{n j}-t_{n k}}{2}\right)}
\end{aligned}
$$


On the other hand, from the hypothesis, we have

$$
\begin{aligned}
\left|t_{n j}-t_{n k}\right| & \geq\left|\frac{2 j \pi}{n+1}-\frac{2 k \pi}{n+1}\right|-\left|t_{n j}-\frac{2 j \pi}{n+1}\right|-\left|t_{n k}-\frac{2 k \pi}{n+1}\right| \\
& \geq \frac{2 \pi|j-k|}{n+1}-\frac{2 \delta_{p}}{n+1} \\
& \geq \frac{2 \pi}{n+1}\left(|j-k|-\frac{1}{4}\right)>\frac{\pi}{n+1}|j-k|
\end{aligned}
$$

for $j \neq k$, by recalling from (2.12) that $\delta_{p} \leq \frac{1}{30}<\frac{\pi}{4}$. Hence, since $\sin \theta \geq$ $2 \theta / \pi$ for $0 \leq \theta \leq \pi / 2$ and, as a function of $x$,

$$
\frac{4 \rho^{2} x}{\left(1-\rho^{2}\right)^{2}+4 \rho^{2} x}
$$

is monotonically increasing for $x \geq 0$, we have for $0 \leq t_{n j}-t_{n k} \leq \pi$ or $-\pi \leq t_{n j}-t_{n k} \leq 0$, by applying (2.15):

$$
\left|\frac{\zeta_{j}-\zeta_{k}}{1-\bar{\zeta}_{k} \zeta_{j}}\right|^{2} \geq \frac{4 \rho^{2}\left(\frac{2}{\pi}\right)^{2}\left(\frac{\pi}{2(n+1)}\right)^{2}(j-k)^{2}}{\left(1-\rho^{2}\right)^{2}+4 \rho^{2}\left(\frac{2}{\pi}\right)^{2}\left(\frac{\pi}{2(n+1)}\right)^{2}(j-k)^{2}}
$$

Consequently, in view of the fact that each term is less than 1 , we may conclude from (2.14) that

$$
\prod_{\substack{j=0 \\ j \neq k}}^{n}\left|\frac{\zeta_{j}-\zeta_{k}}{1-\bar{\zeta}_{k} \zeta_{j}}\right|^{2} \geq \prod_{l=1}^{n}\left(\frac{4 \rho^{2}\left(\frac{2}{\pi}\right)^{2}\left(\frac{\pi}{2(n+1)}\right)^{2} l^{2}}{\left(1-\rho^{2}\right)^{2}+4 \rho^{2}\left(\frac{2}{\pi}\right)^{2}\left(\frac{\pi}{2(n+1)}\right)^{2} l^{2}}\right)^{2} .
$$

To estimate the lower bound in $(2.16)$, we recall that $\rho=\left(1-\frac{1}{n+1}\right)$, so that

$$
\left(1-\rho^{2}\right)^{2} \leq \frac{4}{(n+1)^{2}} \leq \frac{8 \rho^{2}}{(n+1)^{2}}
$$

and (2.16) yields

$$
\prod_{\substack{j=0 \\ j \neq k}}^{n}\left|\frac{\zeta_{j}-\zeta_{k}}{1-\bar{\zeta}_{k} \zeta_{j}}\right| \geq \prod_{l=1}^{n} \frac{l^{2}}{2+l^{2}} \geq \prod_{l=1}^{\infty}\left(1-\frac{2}{2+l^{2}}\right)=: \delta
$$

where $\delta>0$, since $\sum_{l=1}^{\infty} 2 /\left(2+l^{2}\right)<\infty$.

\section{Proof of the theorems}

We are now ready to prove Theorem 1 . For any polynomial $P_{n} \in \pi_{n}$, we use the notation

$$
P_{n}^{*}(z)=P_{n}\left(\left(1-\frac{1}{n+1}\right)^{-1} z\right)
$$

so that

$$
P_{n}^{*}\left(\left(1-\frac{1}{n+1}\right) e^{i t_{n k}}\right)=P_{n}\left(z_{n k}\right)
$$


where $z_{n k}=e^{i t_{n k}}$, and

$$
\begin{aligned}
\int_{|z|=1}\left|P_{n}(z)\right|^{p}|d z| & \leq \int_{|z|=1+\frac{1}{n}}\left|P_{n}(z)\right|^{p}|d z| \\
& =\left(1+\frac{1}{n}\right) \int_{|z|=1}\left|P_{n}^{*}(z)\right|^{p}|d z| .
\end{aligned}
$$

From hypothesis (1.2) on $\mu_{n}$, we can apply Lemma 3 and conclude from Theorem $\mathrm{F}$ that there exists a function $g \in H^{p}$ which satisfies

$$
g\left(\left(1-\frac{1}{n+1}\right) e^{i t_{n k}}\right)=P_{n}^{*}\left(\left(1-\frac{1}{n+1}\right) e^{i t_{n k}}\right)=P_{n}\left(z_{n k}\right)
$$

$k=0, \ldots, n$, by using (3.2), and

$$
\frac{1}{2 \pi} \int_{0}^{2 \pi}\left|g\left(e^{i \theta}\right)\right|^{p} d \theta \leq \frac{2 C_{p, \delta}}{n+1} \sum_{k=0}^{n}\left|P_{n}\left(z_{n k}\right)\right|^{p}
$$

by observing that

$$
1-\left|\left(1-\frac{1}{n+1}\right) e^{i t_{n k}}\right|^{2} \leq \frac{2}{n+1} .
$$

Hence, to complete the proof of Theorem 2, it is sufficient to show that

$$
\int_{0}^{2 \pi}\left|P_{n}\left(e^{i \theta}\right)\right|^{p} d \theta \leq C \int_{0}^{2 \pi}\left|g\left(e^{i \theta}\right)\right|^{p} d \theta
$$

for some absolute constant $C$. Now, from (3.4), it is well known (cf. [17, Chapter 3]) that

$$
g(z)-P_{n}^{*}(z)=\frac{1}{2 \pi i} \int_{|\zeta|=1} \frac{\omega_{n}(z)}{\omega_{n}(\zeta)} \frac{g(\zeta)}{\zeta-z} d \zeta
$$

for $|z|<1$, where $\omega_{n}$ is defined in (2.6). By using the notation in (2.2), we have

$$
\left(\frac{g-P_{n}^{*}}{\omega_{n}}\right)(z)=\left(H\left(\frac{g}{\omega_{n}}\right)\right)(z), \quad|z|<1 .
$$

Also, for $1<p<\infty$ and under the condition (1.2), Lemma 2 allows us to conclude that $\left|\omega_{n}\left(e^{i \theta}\right)\right|^{p}$ is an $A_{p}$-weight. Hence, from Theorem E, it follows that

$$
\begin{aligned}
\int_{0}^{2 \pi}\left|g\left(e^{i \theta}\right)-P_{n}^{*}\left(e^{i \theta}\right)\right|^{p} d \theta & =\int_{0}^{2 \pi}\left|\left(H\left(\frac{g}{\omega_{n}}\right)\right)\left(e^{i \theta}\right)\right|^{p}\left|\omega_{n}\left(e^{i \theta}\right)\right|^{p} d \theta \\
& \leq C_{H} \int_{0}^{2 \pi}\left|g\left(e^{i \theta}\right)\right|^{p} d \theta
\end{aligned}
$$

so that, by Minkowski's inequality,

$$
\int_{0}^{2 \pi}\left|P_{n}^{*}\left(e^{i \theta}\right)\right|^{p} d \theta \leq\left(1+C_{H}^{\frac{1}{p}}\right)^{p} \int_{0}^{2 \pi}\left|g\left(e^{i \theta}\right)\right|^{p} d \theta .
$$

Finally, by applying (3.3) and (3.8), we arrive at (3.6) with $C \leq 2\left(1+C_{H}^{\frac{1}{p}}\right)^{p}$. This completes the proof of Theorem 1. 
To prove Theorem 2, we first remark that the case $0<p \leq 1$ can be reduced to the case $1<p<\infty$ by a simple application of Hölder's inequality (cf. [15]). Hence, we now assume that $1<p<\infty$ and that (1.2) is satisfied. Let $f \in A$ and $\widehat{P}_{n}$ be its best uniform approximant from $\pi_{n}$; that is, $\widehat{P}_{n} \in \pi_{n}$ and

$$
\left\|\widehat{P}_{n}-f\right\|_{\infty}=E_{n}(f) \text {. }
$$

Since $L_{n}\left(\widehat{P}_{n} ; \cdot\right)=\widehat{P}_{n}$, we have

$$
\left\|L_{n}(f ; \cdot)-f\right\|_{p} \leq\left\|\widehat{P}_{n}-f\right\|_{p}+\left\|L_{n}\left(f-\widehat{P}_{n} ; \cdot\right)\right\|_{p} .
$$

Also, since $L_{n}\left(f-\widehat{P}_{n} ; z_{n k}\right)=f\left(z_{n k}\right)-\widehat{P}_{n}\left(z_{n k}\right)$, it follows from Theorem 1 that

$$
\left\|L_{n}\left(f-\widehat{P}_{n} ; \cdot\right)\right\|_{p}^{p} \leq \frac{C_{p}}{n+1} \sum_{k=0}^{n}\left|f\left(z_{n k}\right)-\widehat{P}_{n}\left(z_{n k}\right)\right|^{p} .
$$

Hence, an application of (3.9) and (3.10) yields:

$$
\left\|L_{n}(f ; \cdot)-f\right\|_{p} \leq\left(1+C_{p}^{\frac{1}{p}}\right) E_{n}(f) .
$$

This completes the proof of Theorem 2 with $C_{p}^{\prime}=1+C_{p}^{\frac{1}{p}}$.

\section{DEPENDENCE OF $\delta_{p}$ ON $p$}

In this section, we construct a family $\left\{z_{n k}: k=0, \ldots, n\right\}$ on $T$ with $\mu_{n} \leq \frac{\delta_{2}}{n+1}$ for some constant $\delta_{2}>0$ such that

$$
\left\|L_{n}(f ; \cdot)-f\right\|_{2} \leq C_{2}^{\prime} E_{n}(f)
$$

for all $f \in A$, but

$$
\sup \left\{\left\|L_{n}(f ; \cdot)\right\|_{p}: f \in A,\|f\|_{\infty}=1\right\} \rightarrow \infty
$$

for all sufficiently large values of $p$.

According to Theorem 1, there exists a $\delta_{2}$ with $\frac{2 \pi}{n+1}>\delta_{2}>0$ such that if we select

$$
z_{n k}= \begin{cases}\exp \left(i \frac{2 k \pi+\delta_{2}}{n+1}\right) & \text { for } 0 \leq k \leq\left[\frac{n}{2}\right], \\ \exp \left(i \frac{2 k \pi}{n+1}\right) & \text { for }\left[\frac{n}{2}\right]<k \leq n,\end{cases}
$$

then (4.1) is satisfied. Let $\lambda_{n}(z)=\prod_{k=0}^{n}\left(z-z_{n k}\right)$. Then we may also write

$$
\lambda_{n}(z)=\left(z^{n+1}-1\right) \prod_{k=0}^{\left[\frac{n}{2}\right]} \frac{z-z_{n k}}{z-e^{i \frac{2 k}{n+1}}} .
$$


Hence, for $\frac{5}{8} n \leq j \leq \frac{7}{8} n$, we have

$$
\begin{aligned}
\left|\lambda_{n}^{\prime}\left(z_{n j}\right)\right| & =(n+1)\left|z_{n j}^{n} \prod_{k=0}^{\left[\frac{n}{2}\right]} \frac{z_{n j}-z_{n k}}{z_{n j}-e^{i \frac{2 k n}{n+1}}}\right| \\
& \leq(n+1) \prod_{k=0}^{\left[\frac{n}{2}\right]}\left(1+\left|\frac{1-e^{i \frac{\delta_{2}}{n+1}}}{1-e^{i \frac{2(k-j) \pi}{n+1}}}\right|\right) \\
& \leq(n+1)\left(1+\frac{\frac{\delta_{2}}{n+1}}{2 \sin \frac{\pi}{8}}\right)^{\left[\frac{n}{2}\right]+1} \leq c_{1} n
\end{aligned}
$$

for some constant $c_{1}>0$. This gives

$$
\sum_{k=0}^{n} \frac{1}{\left|\lambda_{n}^{\prime}\left(z_{n k}\right)\right|} \geq \sum_{\frac{5 n}{8} \leq k \leq \frac{7 n}{8}} \frac{1}{\left|\lambda_{n}^{\prime}\left(z_{n k}\right)\right|} \geq \frac{1}{4 c_{1}} .
$$

On the other hand, for $\zeta_{0}=e^{-i \frac{\pi}{2(n+1)}}$, we have, from (4.4),

$$
\left|\frac{-i-1}{\lambda_{n}\left(\zeta_{0}\right)}\right|=\left|\frac{\zeta_{0}^{n+1}-1}{\lambda_{n}\left(\zeta_{0}\right)}\right|=\prod_{k=0}^{\left[\frac{n}{2}\right]}\left|1-\frac{e^{i \frac{2 k \pi}{n+1}}-z_{n k}}{\zeta_{0}-z_{n k}}\right| .
$$

It is obvious that there exists a constant $\varepsilon>0$ such that whenever $0 \leq k \leq \varepsilon n$, we have

$$
\left|\arg \frac{e^{i \frac{2 k \pi}{n+1}}-z_{n k}}{\zeta_{0}-z_{n k}}\right| \leq \frac{\pi}{4}
$$

Hence, it follows that

$$
\begin{aligned}
\prod_{0 \leq k \leq \varepsilon n}\left|1-\frac{e^{i \frac{2 k \pi}{n+1}}-z_{n k}}{\zeta_{0}-z_{n k}}\right| & \leq \prod_{0 \leq k \leq \varepsilon n}|1-| \frac{e^{i \frac{2 k \pi}{n+1}}-z_{n k}}{\zeta_{0}-z_{n k}}\left|e^{i \frac{\pi}{4}}\right| \\
& \leq c_{2} \prod_{1 \leq k \leq \varepsilon n}\left(1-\frac{c_{3} \delta_{2}}{k}\right) \leq c_{2}(\varepsilon n)^{-c_{4} \delta_{2}}
\end{aligned}
$$

for some absolute positive constants $c_{2}, c_{3}$, and $c_{4}$. In addition,

$$
\begin{aligned}
\prod_{\varepsilon n<k<\left[\frac{n}{2}\right]}\left|1-\frac{e^{i \frac{i k \pi}{n+1}}-z_{n k}}{\zeta_{0}-z_{n k}}\right| & \leq \prod_{\varepsilon n<k \leq\left\lfloor\frac{n}{2}\right]}\left(1+\left|\frac{e^{i \frac{2 k \pi}{n+1}}-z_{n k}}{\zeta_{0}-z_{n k}}\right|\right) \\
& \leq \prod_{\varepsilon n<k \leq\left[\frac{n}{2}\right]}\left(1+\frac{c_{5} \delta_{2}}{k}\right) \leq c_{6}
\end{aligned}
$$

for some absolute constants $c_{5}$ and $c_{6}$. By combining the information from (4.6), (4.7), and (4.8), we obtain

$$
\left|\lambda_{n}\left(\zeta_{0}\right)\right| \geq c_{7} n^{c_{4} \delta_{2}}
$$

for some $c_{7}>0$. Hence, using (4.5) and (4.9), we arrive at

$$
\sum_{k=0}^{n}\left|\frac{\lambda_{n}\left(\zeta_{0}\right)}{\left(\zeta_{0}-z_{n k}\right) \lambda_{n}^{\prime}\left(z_{n k}\right)}\right| \geq \frac{c_{7}}{8 c_{1}} n^{c_{4} \delta_{2}}
$$


To get rid of the absolute value, let

$$
\arg \frac{\lambda_{n}\left(\zeta_{0}\right)}{\left(\zeta_{0}-z_{n k}\right) \lambda_{n}^{\prime}\left(z_{n k}\right)}=\theta_{n k}
$$

By a lemma in [3], there exists a function $f_{n} \in A$ satisfying $\left\|f_{n}\right\|_{\infty}=1$ and $f_{n}\left(z_{n k}\right)=e^{-i \theta_{n k}}, k=0, \ldots, n$. Hence, (4.10) yields

$$
\left|L_{n}\left(f_{n} ; \zeta_{0}\right)\right|=\left|\sum_{k=0}^{n} f_{n}\left(z_{n k}\right) \frac{\lambda_{n}\left(\zeta_{0}\right)}{\left(\zeta_{0}-z_{n k}\right) \lambda_{n}^{\prime}\left(z_{n k}\right)}\right| \geq \frac{c_{7}}{8 c_{1}} n^{c_{4} \delta_{2}} .
$$

Finally, by one of the two Marcinkiewicz-Zygmunds inequalities (cf. [20, p. 30]) and (4.11), we have, for $p \geq 1$,

$$
\begin{aligned}
\frac{1}{2 \pi} \int_{0}^{2 \pi}\left|L_{n}\left(f_{n} ; e^{i \theta}\right)\right|^{p} d \theta & \geq \frac{c_{p}^{\prime \prime}}{n+1} \sum_{k=0}^{n}\left|L_{n}\left(f_{n} ; \zeta_{0} e^{i \frac{2 k \pi}{n+1}}\right)\right|^{p} \\
& \geq \frac{c_{p}^{\prime \prime}}{n+1}\left|L_{n}\left(f_{n} ; \zeta_{0}\right)\right|^{p} \geq c_{8} n^{p c_{4} \delta_{2}-1}
\end{aligned}
$$

for some absolute constant $c_{8}$. Hence, using the fact that $\left\|f_{n}\right\|_{\infty}=1$, we arrive at (4.2) for $p>\frac{1}{c_{4} \delta_{2}}$.

\section{FinAl REMARKS}

I. An assumption such as (1.2) on the distribution of $\left\{z_{n k}: k=0, \ldots, n\right\}$ on $T$ is necessary for $\left\|L_{n}(f ; \cdot)-f\right\|_{p} \rightarrow 0$ for all $f \in A$. We already know from Theorem A that for $p=\infty$, this family must necessarily be uniformly distributed on $T$. In the following, we will show that the uniform distribution of $\left\{z_{n k}: k=0, \ldots, n\right\}$ on $T$ is also necessary for $\left\|L_{n}(f ; \cdot)-f\right\|_{p} \rightarrow 0$ for all $f \in A$. Let

$$
\eta_{n}(z)=\prod_{k=0}^{n}\left(z-z_{n k}\right) \text {. }
$$

It is well known (cf. [7, Chapter 2]) that the uniform distribution of the family $\left\{z_{n k}: k=0, \ldots, n\right\}$ on $T$ is equivalent to

$$
\lim _{n \rightarrow \infty} \frac{\left|\eta_{n}(z)\right|^{\frac{1}{n+1}}}{|z|}=1, \quad|z|>1,
$$

where the convergence is uniform on every compact subset in $|z|>1$. Assume that (5.1) does not hold. Then it is known (cf. [17, p. 161]) that there is some $\zeta_{0},\left|\zeta_{0}\right|>1$, and a sequence of integers $n_{k} \rightarrow \infty$ such that $\mid \eta_{n_{k}}\left(\zeta_{0}\right)^{\frac{1}{n_{k}+1}}<1$ for all $k$, so that

$$
\left|\eta_{n_{k}}\left(\zeta_{0}\right)\right|<\left(1-\varepsilon_{0}\right)^{n_{k}+1}
$$

for some $\varepsilon_{0}>0$ and all $k$. Consider the function $f_{0}(z)=1\left(\zeta_{0}-z\right)$ which is in $A$. Then it follows from the formula

$$
f_{0}(z)-L_{n}\left(f_{0} ; z\right)=\frac{\eta_{n}(z)}{\eta_{n}\left(\zeta_{0}\right)\left(\zeta_{0}-z\right)}
$$


that

$$
\left|f_{0}(0)-L_{n_{k}}\left(f_{0} ; 0\right)\right|=\frac{1}{\left|\eta_{n_{k}}\left(\zeta_{0}\right)\right|\left|\zeta_{0}\right|},
$$

which tends to $\infty$ in view of (5.2). Hence, by [5, Theorem 1.5],

$$
\left\|L_{n}\left(f_{0} ; \cdot\right)-f_{0}\right\|_{p} \nrightarrow 0 \text {. }
$$

II. Recall that two of the main tools in establishing Theorem 1 , and hence Theorem 2, are the $H^{p}$-interpolation result stated in Theorem $\mathrm{F}$ and the integral representation formula in equality (3.7). These two results, however, can be generalized to multiple nodes $z_{n k}$. In addition, if each $z_{n k}, k=0, \ldots, n$, has the same multiplicity, then Lemma 2 also applies, since $\left|\omega_{n}\left(e^{i \theta}\right)\right|^{\alpha}$ is always an $A_{p}$-weight for any $\alpha>0$. Hence, for any nonnegative integer $q$, by setting $N=(q+1)(n+1)-1$, a simple modification of our proof of Theorem 1 yields the inequality

$$
\frac{1}{2 \pi} \int_{0}^{2 \pi}\left|P_{N}\left(e^{i \theta}\right)\right|^{p} d \theta \leq \frac{C_{p}}{n+1} \sum_{k=0}^{n} \sum_{j=0}^{q} \frac{\left|P_{N}^{(j)}\left(z_{n k}\right)\right|^{p}}{(n+1)^{j p}}
$$

for all $P_{N} \in \pi_{N}$ provided that (1.2) is satisfied, where $1<p<\infty$. Of course, a different value of $\delta_{p}$ is required. Consequently, Theorem 2 can be easily extended to Hermite or Hermite-Fejer interpolation with the same estimates. In [20], this result was established for the roots of unities using another method.

Of course, the analogous problems for nodes $z_{n k}$ with different multiplicities still remain open.

III. A seemingly very difficult problem is to determine the largest $\delta_{p}$ in the condition (1.2) for the validity of Theorems 1 and 2.

IV. A more interesting problem is to find a necessary and sufficient condition on the distribution of $\left\{z_{n k}: k=0, \ldots, n\right\}$ so that $\left\|L_{n}(f ; \cdot)-f\right\|_{p} \rightarrow 0$ for all $f \in A$, where $0<p<\infty$. Recall that (1.2) is a sufficient condition and the uniform distribution on $T$ is a necessary condition. We remark that an example can be constructed to show that the uniform distribution of $\left\{z_{n k}: k=\right.$ $0, \ldots, n\}$ on $T$ is not sufficient for $L_{p}$ convergence, $0<p<\infty$.

\section{REFERENCES}

1. S. Ja. Al'per and G. I. Kalinogorskaja, The convergence of Lagrange interpolating polynomials in the complex domain, Izv. Vyssh. Uchebn. Zaved. Math. 11(90) (1969), 12-23.

2. C. K. Chui and X. C. Shen, On Hermite-Fejér interpolation in a Jordan domain, Trans. Amer. Math. Soc. 323 (1991), 93-109.

3. J. G. Clunie and J. C. Mason, Norms of analytic interpolation projections on a general domains, J. Approx. Theory 41 (1984), 149-158.

4. J. H. Curtiss, Convergence of complex Lagrange interpolation polynomial on the locus of the interpolation points, Duke Math. J. 32 (1965), 187-204.

5. P. L. Duren, Theory of $H^{p}$ space, Academic Press, New York, 1970.

6. L. Fejér, Über Interpolation, Göttlinger Nachr. (1916), 66-91.

7. D. Gaier, Vorlesungen Über Approximation in Komplexens, Birkhäuser Verlag, Basel, Boston, and Stuttgart, 1980.

8. J. B. Garnett, Bounded analytic functions, Academic Press, New York, 1981.

9. P. Koosis, Introduction to $H^{p}$ spaces, London Math. Soc. Lecture Note Ser., no. 40, Cambridge Univ. Press, Cambridge, 1980. 
10. S. M. Lozinski, Über Interpolation, Mat. Sb. 8(50) (1941), 57-58.

11. B. Muckenhoupt, Weight norm inequalities for Hardy maximal functions, Trans. Amer. Math. Soc. 165 (1972), 207-226.

12. E. B. Saff and J. L. Walsh, On the convergence of rational functions which interpolate in the roots of unity, Pacific J. Math. 45 (1973), 639-650.

13. A. Sharma and P. Vértesi, Mean convergence and interpolation in roots of unity, SIAM J. Math. Anal. 14 (1983), 800-806.

14. X. C. Shen, The convergence of $(0,1, \ldots, q)$ Hermite-Fejér interpolating polynomials on the roots of unity, Chinese Ann. Math. Ser. (to appear).

15. X. C. Shen and L. F. Zhong, Approximation in the mean by Lagrange interpolating polynomials in the complex plane, Kuxue Tongbao (A Monthly of Sciences) 33 (1988), 819-824.

16. P. Vértesi, On the almost everywhere divergence of Lagrange interpolation (complex and trigonometric cases), Acta Math. Acad. Sci. Hungar. 39 (1982), 367-377.

17. J. L. Walsh, Interpolation and approximation by rational functions in the complex domain, Amer. Math. Soc. Colloq. Publ., Vol. 20, Amer. Math. Soc., Providence, R. I., 1960.

18. J. L. Walsh and A. Sharma, Least squares and interpolation in roots of unity, Pacific J. Math. 14 (1964), 727-750.

19. Y. Xu, The generalized Marcinkiewicz-Zygmund inequality for trigonometric polynomials, J. Math. Anal. Appl. (to appear).

20. A. Zygmund, Trigonometric series, Vol. II, Cambridge Univ. Press, Cambridge, 1959.

(C. K. Chui) Department of Mathematics, Texas A\&M University, College Station, TEXAS 77843

E-mail address: centrap@tamvm1.bitnet

(C.-C. Shen (deceased) and L. Zhong) Department of Mathematics, Peking University, Beijing, People's Republic of China 